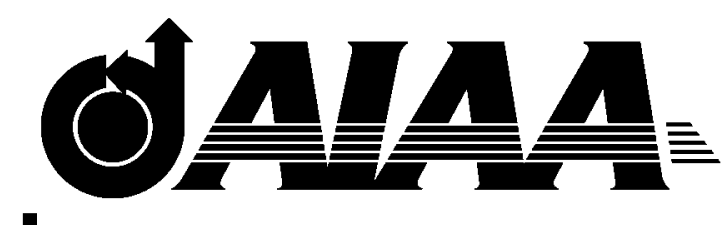

\title{
AIAA 2002-4759 \\ LPV Controller Interpolation for Improved Gain-Scheduling Control Performance
}

Fen Wu

Dept. of Mechanical and Aerospace Engineering

North Carolina State University

Raleigh, NC 27695

SungWan Kim

Dynamics and Control Branch

NASA Langley Research Center

Hampton, VA 23681-2199

\section{AIAA Guidance, Navigation, and Control Conference August 5-8, 2002/Monterey, CA}




\title{
LPV Controller Interpolation for Improved Gain-Scheduling Control Performance
}

\author{
Fen $\mathrm{Wu}^{*}$ \\ Dept. of Mechanical and Aerospace Engineering \\ North Carolina State University \\ Raleigh, NC 27695 \\ SungWan Kim ${ }^{\dagger}$ \\ Dynamics and Control Branch \\ NASA Langley Research Center \\ Hampton, VA 23681-2199
}

In this paper, a new gain-scheduling control design approach is proposed by combining LPV control theory with interpolation techniques. The improvement of gain-scheduled controllers can be achieved from local synthesis of Lyapunov functions and continuous construction of a global Lyapunov function by interpolation. It has been shown that this combined LPV control design scheme is capable of improving closed-loop performance derived from local performance improvement. The gain of the LPV controller will also change continuously across parameter space. The advantages of the newly proposed LPV control is demonstrated through a detailed AMB controller design example.

\section{Introduction}

The gain-scheduling approach is perhaps one of the most popular nonlinear control design techniques that has been widely used in fields ranging from aerospace to process control. Although it seems to work well in practice, this heuristic design procedure does not take the parameter variations into account. 1,2 In its early practice, the control design came with virtually no guarantee on performance, robustness, or even nominal stability. Recently, a systematic gain-scheduling design technique was developed in the form of linear parameter-varying (LPV) control theory. ${ }^{3-8}$ This class of systems is different from the standard linear timevarying counterpart due to the causal dependence of its controller gains on the variations of the plant $d y-$ namics. The implications of parameter-dependent systems theory for gain scheduling is obvious because gain scheduling conceptually involves a linear, parameterdependent plant. The LPV design technique provides

\footnotetext{
*Assistant Professor, AIAA Member. E-mail: fwu@eos.ncsu.edu, Phone: (919) 515-5268, Fax: (919) 515-7968. This research was supported by Grant from NASA Langley Research Center (NAG-1-01119).

'Research Engineer, AIAA Senior Member.

Copyright (C) 2002 by Fen Wu. Published by the American Institute of Aeronautics and Astronautics, Inc. with permission.
}

guaranteed stability and performance properties, and simplifies the interpolation and realization problems associated with conventional gain-scheduling methods. However, one potential problem associated with parameter-dependent Lyapunov function approach is the lack of guidance for choosing "right" basis functions to parameterize infinite dimensional functional space.

Interpolation is an important step toward synthesis of gain-scheduled controllers and has not been adequately addressed in a systematic way. Some ad-hoc interpolation techniques have been proposed in the past: (1) linear interpolation of poles, zeros, and gains of local controllers; (2) linear interpolation of solutions of Riccati equations; and (3) linear interpolation of state-space matrices of balanced controller realizations. These approaches are intuitively appealing but could generate destabilizing controllers. Stilwell and Rugh ${ }^{9}$ proposed a gain-scheduled control design based on interpolation techniques. The interpolated parameter-varying controller preserves point-wise stability of local LTI controllers for all frozen parameter values. However, the global control does not provide a priori stability and performance guarantee from locally designed controllers for fast time-varying parameters.

In this research, we propose an interpolating LPV control approach by combining LPV control theory with interpolation techniques. The interpolated LPV control design is capable of improving controlled performance by finding the most appropriate Lyapunov functions in a local sense. Moreover, the local stability property will be extended to the entire parameter range by a globally constructed Lyapunov function through interpolation. The proposed interpolating LPV control technique has great potential to many industrial applications including active magnetic bearings (AMBs), for which the controller adjusts its gain based on changing rotor speed and provides accurate control of flexible mode variation in terms of natural

1 OF 9 
frequency, damping, and mode shape. In this research, we use a simple rigid magnetic bearing example to demonstrate advantages over existing gain-scheduling approaches.

\section{Improved LPV Analysis Condition}

Consider an LPV system

$$
\left[\begin{array}{l}
\dot{x}(t) \\
e(t)
\end{array}\right]=\left[\begin{array}{ll}
A(\rho(t)) & B(\rho(t)) \\
C(\rho(t)) & D(\rho(t))
\end{array}\right]\left[\begin{array}{l}
x(t) \\
d(t)
\end{array}\right]
$$

where $x, \dot{x} \in \mathbf{R}^{n}, d \in \mathbf{R}^{n_{d}}, e \in \mathbf{R}^{n_{e}}$. All the matrices have compatible dimensions. It is assumed that the vector-valued parameter $\rho$ evolves continuously over time and its range is limited to a compact subset $\mathcal{P} \subset \mathbf{R}^{s}$. Its time derivative is bounded and satisfies the constraint $-\nu_{i} \leq \dot{\rho}_{i} \leq$ $\nu_{i}, i=1,2, \cdots, s$. For notational purposes, $\mathcal{V}:=$ $\left\{v:-\nu_{i} \leq v_{i} \leq \nu_{i}, i=1,2, \cdots, s\right\}$, where $\mathcal{V}$ is a given convex polytope in $\mathbf{R}^{s}$ that contains the origin. Given the sets $\mathcal{P}$ and $\mathcal{V}$, we define the parameter $\nu$-variation set as

$\mathcal{F}_{\mathcal{P}}^{\nu}=\left\{\rho \in \mathcal{C}^{1}\left(\mathbf{R}_{+}, \mathbf{R}^{s}\right): \rho(t) \in \mathcal{P}, \dot{\rho}(t) \in \mathcal{V}, \forall t \geq 0\right\}$

Therefore the dynamics of the LPV system are characterized by the parameter value $\rho$ and its variation along time. Previous research on LPV control theory mainly focused on a single Lyapunov function (quadratic or parameter-dependent) over the entire parameter set. For the given LPV system, it is clear that the achievable performance relies on the choice of the Lyapunov function. However, it would be beneficial if one can analyze the performance of the LPV system over different parameter ranges using different Lyapunov functions with a stability guarantee.

To simplify the presentation, let us assume that the parameter set has dimension one. That is, $\mathcal{P} \subset \mathbf{R}^{1}$. Suppose $\mathcal{P}_{1}, \mathcal{P}_{2}$ is an overlapped partition of the parameter set $\mathcal{P}$, and define the intersection of $\mathcal{P}^{1}$ and $\mathcal{P}^{2}$ as

$$
\mathcal{P}^{12}=\mathcal{P}^{1} \cap \mathcal{P}^{2}
$$

Then the parameter space $\mathcal{P}$ is the union of two subsets $\mathcal{P}^{1}, \mathcal{P}^{2}$. Through linear interpolation of the Lyapunov functions $X_{1}(\rho), X_{1}(\rho)$ over subsets $\mathcal{P}^{1}$ and $\mathcal{P}^{2}$, one would obtain a continuous Lyapunov function over the entire parameter space. The continuity property of the Lyapunov function is important to guarantee monotonic decrease of its value over any allowable trajectories dictated by the LPV system dynamics. A new LPV stability and performance analysis condition based on the partitioned parameter subsets is then proposed.

Theorem 1 For the parameter set $\mathcal{P}=[\rho, \bar{\rho}]$ with its overlapped partition $\mathcal{P}^{1}=[\rho, b]$ and $\mathcal{P}^{2}=[a, \bar{\rho}]$ $(a<b)$, the $L P V$ system (1), and a given symmetric matrix function $Q_{X}(\rho) \geq 0$, if there exists a group of continuously differentiable positive-definite matrix functions $X_{i}(\rho), i=1,2$ such that for any $\rho \in \mathcal{P}^{i}$,

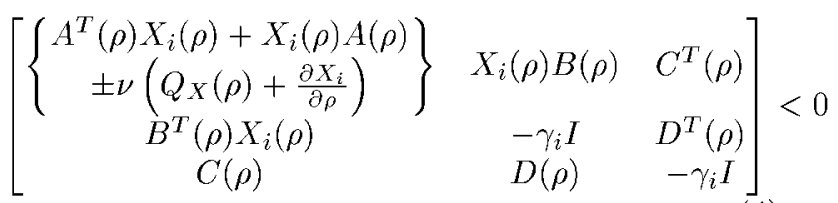

and for all $\rho \in \mathcal{P}^{12}$

$$
\begin{aligned}
& X_{2}(\rho)-X_{1}(\rho)+(b-a) Q_{X}(\rho) \geq 0 \\
& X_{2}(\rho)-X_{1}(\rho)-(b-a) Q_{X}(\rho) \leq 0
\end{aligned}
$$

then the LPV system (1) is exponentially stable and its induced $\mathcal{L}_{2}$ norm with $x(0)=0$ is bounded by $\gamma=$ $\max \left\{\gamma_{1}, \gamma_{2}\right\}$.

Given the above conditions over each parameter subset, a continuous Lyapunov function in the form of

$$
V(x, \rho)=x^{T}\left[\left(\frac{b-\rho}{b-a}\right) X_{1}(\rho)+\left(\frac{\rho-a}{b-a}\right) X_{2}(\rho)\right] x
$$

can be constructed and used to verify the desired stability and performance properties of the system for any parameter trajectories within $\mathcal{F}_{\mathcal{P}}^{\nu}$. Then a non-smooth dissipative system theory is applied to address possible discontinuity of the Lyapunov function derivative at the boundary of the parameter subsets. ${ }^{10}$ The performance bound derived in Theorem 1 states the "worstcase" performance, which is not often achievable. For example, if the parameter trajectory is constrained in the subset $\mathcal{P}^{i}$, then the system's performance is overbounded by $\gamma_{i}$, which could be less than $\max \left\{\gamma_{1}, \gamma_{2}\right\}$.

Theoretically, this theorem simply states the fact that the performance level of the LPV system relates to the existence of a continuous Lyapunov function. However, in practical situations, it is usually hard to identify a suitable parameter-dependent Lyapunov function without resorting to a global search. In this sense, the improved analysis condition will be helpful to find a sharper performance bound through local studies. Then the global Lyapunov function will be constructed by interpolation.

\section{Interpolating LPV Control Synthesis}

Given a generalized open-loop LPV system

$$
\left[\begin{array}{c}
\dot{x}(t) \\
e(t) \\
y(t)
\end{array}\right]=\left[\begin{array}{ccc}
A(\rho(t)) & B_{1}(\rho(t)) & B_{2}(\rho(t)) \\
C_{1}(\rho(t)) & D_{1 \mathbf{1}}(\rho(t)) & D_{12}(\rho(t)) \\
C_{2}(\rho(t)) & D_{21}(\rho(t)) & D_{22}(\rho(t))
\end{array}\right]\left[\begin{array}{c}
x(t) \\
d(t) \\
u(t)
\end{array}\right]
$$

where all of the state-space data are continuous functions of the scheduling parameter $\rho$, it is assumed that the parameter trajectory resides in the set $\mathcal{F}_{\mathcal{P}}^{\nu}$. For simplicity, we assume that 
(A1) $\left(A(\rho), B_{2}(\rho), C_{2}(\rho)\right)$ is parameter-dependently stabilizable and detectable for all $\rho$,

(A2) $D_{12}(\rho)$ and $D_{21}(\rho)$ have full column and row rank respectively for all $\rho$,

(A3) $D_{11}(\rho)=0$ and $D_{22}(\rho)=0$.

We consider the class of interpolating LPV controller in the form of

$$
\left[\begin{array}{c}
\dot{x}_{k}(t) \\
u(t)
\end{array}\right]=\left[\begin{array}{cc}
A_{k}(\rho(t), \dot{\rho}(t)) & B_{k}(\rho(t), \dot{\rho}(t)) \\
C_{k}(\rho(t), \dot{\rho}(t)) & D_{k}(\rho(t), \dot{\rho}(t))
\end{array}\right]\left[\begin{array}{c}
x_{k}(t) \\
y(t)
\end{array}\right]
$$

In general, the controller gain is a continuous function of parameter $\rho$ and its derivative. The control design objective is to minimize the energy $\left(\mathcal{L}_{2}\right.$ norm) of the output signal $e(t)$ of the closed-loop system in the presence of bounded energy disturbance $d(t)$. Specifically for the magnetic bearing control problem, we would like to synthesize a gain-scheduled control with combined disturbance rejection, gyroscopic compensation, and automatic balancing capability. This can be formulated as an optimization problem with minimization of displacement of the rotor from its centerline at selected points subject to unknown torque disturbances.

Define $x_{c l}^{T}:=\left[\begin{array}{ll}x^{T} & x_{k}^{T}\end{array}\right]$, then the closed-loop system can be written as

$$
\left[\begin{array}{c}
\dot{x}_{c l} \\
e
\end{array}\right]=\left[\begin{array}{ll}
A_{c l}(\rho, \dot{\rho}) & B_{c l}(\rho, \dot{\rho}) \\
C_{c l}(\rho, \dot{\rho}) & D_{c l}(\rho, \dot{\rho})
\end{array}\right]\left[\begin{array}{c}
x_{c l} \\
d
\end{array}\right]
$$

where

$$
\begin{gathered}
{\left[\begin{array}{ll}
A_{c l} & B_{c l} \\
C_{c l} & D_{c l}
\end{array}\right]=\left[\begin{array}{cc|c}
A & 0 & B_{1} \\
0 & 0 & 0 \\
\hline C_{1} & 0 & D_{11}
\end{array}\right]} \\
+\left[\begin{array}{cc}
0 & B_{2} \\
I & 0 \\
\hline 0 & D_{12}
\end{array}\right]\left[\begin{array}{cc}
A_{k} & B_{k} \\
C_{k} & D_{k}
\end{array}\right]\left[\begin{array}{cc|c}
0 & I & 0 \\
C_{2} & 0 & D_{21}
\end{array}\right]
\end{gathered}
$$

Next, we propose a synthesis condition for an improved LPV controller using multiple parameterdependent Lyapunov functions and an interpolation scheme. For clarity, only one parameter is considered and the parameter space $\mathcal{P}$ is covered by two overlapped subsets $\mathcal{P}^{1}$ and $\mathcal{P}^{2}$. For each parameter subset, we seek to design one LPV controller as stated in the form of eqn. (9). The overall gain-scheduling controller is then constructed by interpolating local LPV controllers. It is clear that the global controller is capable of achieving tighter performance due to smaller parameter range. However, a critical issue associated with the proposed controller interpolation scheme is the stability of the global LPV controller. This will be guaranteed by constructing a globally continuous Lyapunov function over the entire parameter set.
Theorem 2 For a partition of parameter space $\mathcal{P}=$ $[\rho, \bar{\rho}]$ as subsets $\mathcal{P}^{1}=[\rho, b]$ and $\mathcal{P}^{2}=[a, \bar{\rho}](a<b)$, and given $Q_{R}, Q_{S} \geq 0$ matrix functions, if one of the following equivalent conditions are satisfied:

1. there exist continuously differentiable matrix functions $R_{i}(\rho), S_{i}(\rho), i=1,2$ such that for $\rho \in \mathcal{P}^{i}$

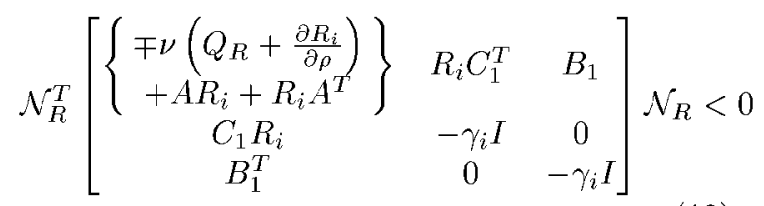

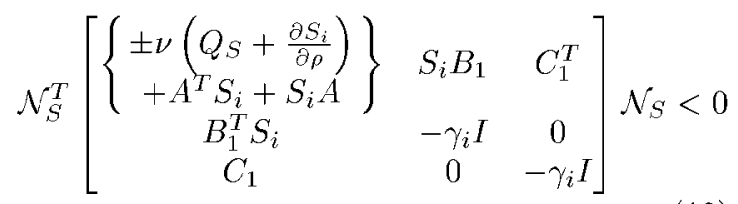

$\left[\begin{array}{cc}R_{i}(\rho) & I \\ I & S_{i}(\rho)\end{array}\right] \geq 0$

with $\mathcal{N}_{R}(\rho)=\operatorname{Ker}\left[B_{2}^{T}(\rho) \quad D_{12}^{T}(\rho) \quad 0\right], \mathcal{N}_{S}(\rho)=$ $\operatorname{Ker}\left[\begin{array}{lll}C_{2}(\rho) & D_{21}(\rho) & 0\end{array}\right]$, and for $\rho \in \mathcal{P}^{12}$

$$
\begin{aligned}
& R_{2}(\rho)-R_{1}(\rho)+(b-a) Q_{R} \geq 0 \\
& R_{2}(\rho)-R_{1}(\rho)-(b-a) Q_{R} \leq 0 \\
& S_{2}(\rho)-S_{1}(\rho)+(b-a) Q_{S} \geq 0 \\
& S_{2}(\rho)-S_{1}(\rho)-(b-a) Q_{S} \leq 0 .
\end{aligned}
$$

2. there exist continuously differentiable matrix functions $R_{i}(\rho), S_{i}(\rho)>0, i=1,2$ and continuous matrix functions $\hat{A}_{k}^{(i)}, \hat{B}_{k}^{(i)}, \hat{C}_{k}^{(i)}, i=1,2$ such that for $\rho \in \mathcal{P}^{i}$,

$$
\begin{aligned}
& {\left[\begin{array}{c} 
\pm \nu\left(Q_{S}+\frac{d S_{i}}{d \rho}\right) \\
+S_{i} A+\hat{B}_{k}^{(i)} C_{2}+(\star)
\end{array}\right\}} \\
& \hat{A}_{k}^{(i) T} \quad\left\{\begin{array}{c}
\mp \nu\left(Q_{R}+\frac{d R_{i}}{d \rho}\right) \\
+A R_{i}+B_{2} \hat{C}_{k}^{(i)}+(\star)
\end{array}\right\} \\
& \begin{array}{c}
B_{1}^{T} S_{i}+D_{21}^{T} \hat{B}_{k}^{(i) T} \\
C_{1}
\end{array} \\
& B_{1}^{T} \\
& C_{1} R_{i}+D_{12} \hat{C}_{k}^{(i)} \\
& S_{i} B_{1}+\hat{B}_{k}^{(i)} D_{21} \\
& \begin{array}{c}
B_{1} \\
-\gamma_{i} I
\end{array} \\
& 0 \\
& \left.\begin{array}{c}
C_{1}^{T} \\
R_{i} C_{1}^{T}+\hat{C}_{k}^{(i) T} D_{12}^{T} \\
0 \\
-\gamma_{i} I
\end{array}\right]<0
\end{aligned}
$$

$\left[\begin{array}{cc}R_{i}(\rho) & I \\ I & S_{i}(\rho)\end{array}\right] \geq 0$

and for $\rho \in \mathcal{P}^{12}$

$$
\begin{aligned}
& R_{2}(\rho)-R_{1}(\rho)+(b-a) Q_{R} \geq 0 \\
& R_{2}(\rho)-R_{1}(\rho)-(b-a) Q_{R} \leq 0 \\
& S_{2}(\rho)-S_{1}(\rho)+(b-a) Q_{S} \geq 0 \\
& S_{2}(\rho)-S_{1}(\rho)-(b-a) Q_{S} \leq 0,
\end{aligned}
$$


then the closed-loop LPV system can be stabilized by a continuous $L P V$ controller with induced $\mathcal{L}_{2}$ performance less than $\bar{\gamma}=\max \left\{\gamma_{1}, \gamma_{2}\right\}$.

Furthermore, let

$$
\begin{gathered}
\gamma(\rho)= \begin{cases}\gamma_{1} \\
\left(\frac{b-\rho}{b-a}\right) \gamma_{1}+\left(\frac{\rho-a}{b-a}\right) \gamma_{2} & \rho \leq a \\
\gamma_{2} & \rho \geq b\end{cases} \\
R(\rho), S(\rho)= \begin{cases}R_{1}(\rho), S_{1}(\rho) & \rho \leq a \\
\left(\frac{b-\rho}{b-a}\right)\left\{R_{1}(\rho), S_{1}(\rho)\right\} & a<\rho<b \\
+\left(\frac{\rho-a}{b-a}\right)\left\{R_{2}(\rho), S_{2}(\rho)\right\} & \rho \geq b \\
R_{2}(\rho), S_{2}(\rho) & \rho\end{cases}
\end{gathered}
$$

and $M(\rho) N^{T}(\rho):=I-R(\rho) S(\rho)$, calculate

$$
\begin{aligned}
& \hat{A}_{k}(\rho, \dot{\rho})=S(\rho) \frac{d R}{d t}+N(\rho) \frac{d M^{T}}{d t}-A^{T}(\rho) \\
& \quad-\frac{1}{\gamma(\rho)}\left[S(\rho) B_{1}(\rho)+\hat{B}_{k}(\rho) D_{21}(\rho) \quad C_{1}^{T}(\rho)\right] \\
& \quad \times\left[B_{1}^{T}(\rho)\right. \\
& \hat{B}_{k}(\rho)=-\left[\gamma(\rho) C_{2}^{T}(\rho)+S(\rho) B_{1}(\rho) D_{21}^{T}(\rho)\right] \\
& \quad \times\left[D_{21}(\rho) D_{21}^{T}(\rho)\right]^{-1} \\
& \hat{C}_{k}(\rho)=-\left[D_{12}^{T}(\rho) D_{12}(\rho)\right]^{-1} \\
& \quad \times\left[\gamma(\rho) B_{2}^{T}(\rho)+D_{12}^{T}(\rho) C_{1}(\rho) R(\rho)\right] \\
& \hat{D}_{k}(\rho)=0
\end{aligned}
$$

Then the interpolated $L P V$ controller $K_{\mathcal{P}}$ will be constructed as

$$
\begin{array}{r}
A_{k}(\rho, \dot{\rho})=N^{-1}(\rho)\left\{\hat{A}_{k}(\rho, \dot{\rho})-S(\rho) B_{2}(\rho) \hat{C}_{k}(\rho)\right. \\
\left.-\hat{B}_{k}(\rho) C_{2}(\rho) R(\rho)-S(\rho) A(\rho) R(\rho)\right\} M^{-T}(\rho)
\end{array}
$$

$$
\begin{aligned}
& B_{k}(\rho)=N^{-1}(\rho) \hat{B}_{k}(\rho) \\
& C_{k}(\rho)=\hat{C}_{k}(\rho) M^{-T}(\rho) \\
& D_{k}(\rho)=0 .
\end{aligned}
$$

It is noted that we ask for different performance levels over each parameter subregion. The proposed interpolation scheme could provide a global stabilizing LPV controller with the potential to improve its local performance. The global Lyapunov function for the closed-loop LPV system is derived from the matrix functions $R_{i}(\rho)$ and $S_{i}(\rho)$. Because of the interpolation scheme used, it relaxes continuity requirement of individual Lyapunov functions over the intersections of parameter subsets. In particular, only finite variation of Lyapunov functions over intersected regions is necessary and the amount of allowable change depends on the maximal parameter variation. When $\nu \rightarrow 0$ (slowly varying case), the conditions (16)-(18) will have no effect on the synthesis result. Thus the synthesis condition over each subset is essentially decoupled and can be solved independently.

The solvability conditions in eqns. (16)-(18) are clearly infinite-dimensional, as is the solution space. To approximate, we restrict the search of the parameter-dependent Lyapunov function to a span of finite numbers of basis functions. That is, let

$$
R_{i}(\rho)=\sum_{k=1}^{N_{f}} f_{i}^{k}(\rho) R_{i}^{k}, \quad S_{i}(\rho)=\sum_{k=1}^{N_{g}} g_{i}^{k}(\rho) S_{i}^{k}
$$

where $\left\{f_{i}^{k}(\rho)\right\}_{k=1}^{N_{f}}$ and $\left\{g_{i}^{k}(\rho)\right\}_{k=1}^{N_{g}}$ are user-specified scalar basis functions. Note that different basis functions may be used over different parameter subsets. $R_{i}^{k}, S_{i}^{k}$ are new optimization variables to be determined. After such a parameterization, the LPV synthesis conditions can be solved using a gridding method over each parameter subset.

\section{Advanced AMB Gain-Scheduling Control}

Active magnetic bearings (AMBs) use an electromagnetic force to provide noncontact support for rotors in high-speed rotating machinery. AMBs have several unique characteristics that make them well suited for aerospace applications: they provide noncontacting support of the flywheel rotor, virtually eliminating bearing friction and wear, and they eliminate concerns with lubrication, heating, and power consumption typical of standard bearing systems. Additionally, AMBs can effectively eliminate synchronous vibrations associated with mass imbalance and shaft run out, making them highly desirable for spacecraft applications where pointing accuracy is critical. Magnetic bearings are well suited as flywheels in replacement of chemical batteries to store energy on a spacecraft, ${ }^{11}$ and they provide integrated attitude control and momentum management functionality. ${ }^{12-14}$ Although this technique has tremendous potential for a variety of industrial applications, AMBs are open-loop unstable, thus making the controller design problem a challenge. Moreover, the flexibility of high-speed rotors adds to the complexity of control design task.

Most controllers in use today for magnetic bearings were designed using PID strategies. However, it is difficult to satisfy the stringent performance requirements with PID control. Mohamed and BuschVishmiac $^{15}$ and Matsumura et al. ${ }^{16}$ designed gainscheduled $\mathcal{H}_{\infty}$ controllers for rigid magnetic bearings utilizing the stabilizing controller parameterization with the free parameter $Q$ playing the scheduling mechanisms. Unfortunately, this ad-hoc gain- 
scheduling design technique suffers from slow varying parameter requirements. The resulting gainscheduling controller could render the closed-loop system unstable when the rotor speed changes rapidly. ${ }^{16}$ Rotor flexibility was considered using the $\mu$-synthesis technique. ${ }^{17}$ It was shown that the $\mu$ controller exhibited much greater stability robustness to variations in rotor mass. However, the controller caused significant performance degradation when the actual rotor speed differs from the pre-specified design condition. One way to address this problem is to design a series of controllers for each operating speed and then interpolate between these controllers. ${ }^{16}$ However, this approach does not provide any stability and performance guarantee. In Tsiotras and Mason ${ }^{18}$ and Tsiotras and Knospe, ${ }^{19}$ the LPV control theory was applied to address variable rotor operating speeds using a rigid rotor model and conservatism of LPV control design was recognized. Similar controllers were also developed by Sivrioglu and Nonami. ${ }^{20}$

\section{Rotor Dynamics Modeling}

Owing to the linear dependence of the rotor speed in the plant dynamics, the nonlinear gyroscopic equations of $\mathrm{AMB}$ can be simplified to a set of linear, time-varying differential equations. However, the rotor dynamics are inherently unstable; that is, even small unbalanced masses can create large synchronous disturbances with the same frequency as the rotor's. Therefore it is necessary to develop a gain-scheduled controller capable of rejecting the periodic disturbance as the rotor speed changes.

The rotational motion of a magnetic bearing can be derived from its rigid body dynamics, which is ${ }^{15,18}$

$$
\begin{aligned}
& \ddot{\theta}=-\frac{\rho J_{a}}{J_{r}} \dot{\psi}+\frac{\ell}{J_{r}}\left(f_{r 1}-f_{r 2}+f_{\ell 2}-f_{\ell 1}+f_{d \theta}\right) \\
& \ddot{\psi}=\frac{\rho J_{a}}{J_{r}} \dot{\theta}+\frac{\ell}{J_{r}}\left(f_{r 3}-f_{r 4}+f_{\ell 4}-f_{\ell 3}+f_{d \psi}\right)
\end{aligned}
$$

where $\theta, \psi$ are the Euler angles denoting the orientation of the rotor centerline. $J_{a}, J_{r}$ are the moment of inertia of the rotor in axial and radial directions, respectively. The parameter $\rho$ denotes the rotor speed. The magnetic forces generated by four pairs of electromagnets are denoted by $f_{r i}, f_{\ell i}$ for $i=1,2,3,4$. $f_{d \theta}, f_{d \psi}$ are disturbance forces caused by gravity, modeling errors, imbalances, etc.

The electromagnetic force $f_{j}$ is related to the voltage $e_{j}$ across the $j$ th coil through the magnetic flux $\psi_{j}$ by the equations

$$
\begin{array}{r}
f_{j}=k \phi_{j}^{2}\left(1+\frac{2 g_{j}}{\pi h}\right) \\
e_{j}=N \frac{d \phi_{j}}{d t}+\frac{2 R}{\nu_{0} A N} g_{j} \phi_{j}
\end{array}
$$

The following system parameters are chosen for the active magnetic bearing example (Table 1).

\begin{tabular}{c|c}
\hline Parameter & Value \\
\hline$A$ area of each pole & $1531.79 \mathrm{~mm}^{2}$ \\
$h$ pole width & $40.00 \mathrm{~mm}$ \\
$G_{0}$ nominal gap & $0.55 \mathrm{~mm}$ \\
$J_{r}$ radial moment of inertia & $0.333 \mathrm{~kg} \cdot \mathrm{m}^{2}$ \\
$J_{a}$ axial moment of inertia & $0.0136 \mathrm{~kg} \cdot \mathrm{m}^{2}$ \\
$\ell$ half the length of the shaft & $0.13 \mathrm{~m}$ \\
$k$ & $4.6755576 \times 10^{8}$ \\
$N$ number of coil turns & 400 \\
$R$ coil resistance & $14.6 \mathrm{Ohm}$ \\
$\Phi_{0}$ nominal airgap & $2.09 \times 10^{-4} \mathrm{~Wb}$ \\
\hline
\end{tabular}

Table 1 Magnetic bearing parameters.

In the absence of disturbances and modeling errors, the above equation specifies an equilibrium. Linearizing the nonlinear equations at the equilibrium, we obtain

$$
\begin{aligned}
\ddot{\theta} & =-\frac{\rho J_{a}}{J_{r}} \dot{\psi}+\frac{\ell}{J_{r}}\left(-4 c_{2} \ell \theta+2 c_{1} \phi_{\theta}+f_{d \theta}\right) \\
\ddot{\psi} & =\frac{\rho J_{a}}{J_{r}} \dot{\theta}+\frac{\ell}{J_{r}}\left(-4 c_{2} \ell \psi+2 c_{1} \phi_{\psi}+f_{d \psi}\right) \\
N \dot{\phi}_{\theta} & =e_{\theta}+2 d_{2} \ell \theta-d_{1} \phi_{\theta} \\
N \dot{\phi}_{\psi} & =e_{\psi}+2 d_{2} \ell \psi-d_{1} \phi_{\psi}
\end{aligned}
$$

where $\phi_{\theta}, \phi_{\psi}$ are the differential magnetic flux from electromagnetic pairs, and $e_{\theta}, e_{\psi}$ are the corresponding differences of electric voltage. The constants $c_{1}, c_{2}, d_{1}, d_{2}$ depend on $\Phi_{0}, G_{0}, R, A, N, \nu_{0}$ and the geometry of the bearings as follows

$$
\begin{aligned}
& c_{1}=2 k \Phi_{0}\left(1+\frac{2 G_{0}}{\pi h}\right), \quad c_{2}=\frac{2 k \Phi_{0}^{2}}{\pi h}, \\
& d_{1}=\frac{2 R G_{0}}{\nu_{0} A N}, \quad d_{2}=\frac{2 R \Phi_{0}}{\nu_{0} A N}, \quad m=\frac{J_{r}}{\ell} .
\end{aligned}
$$

The imbalance forces $f_{d \theta}$ and $f_{d \psi}$ are typically modeled as sinusoidal disturbances, and are given by

$$
\begin{gathered}
f_{d \theta}=\frac{\left(J_{r}-J_{a}\right)}{\ell} \rho^{2} \tau \cos (\rho t), \\
f_{d \psi}=\frac{\left(J_{r}-J_{a}\right)}{\ell} \rho^{2} \tau \sin (\rho t) .
\end{gathered}
$$

In automatic balancing design, the imbalance forces will be treated as a sinusoidal sensor noise $n^{T}=$ $[\ell \tau \sin (\rho t+\lambda) \quad \ell \tau \cos (\rho t+\lambda)]$ on the measured rotor displacement, which can be written in a state-space form of

$$
\begin{aligned}
\dot{x}_{d i} & =\left[\begin{array}{cc}
\sigma & -\rho \\
\rho & \sigma
\end{array}\right] x_{d i}+\left[\begin{array}{l}
1 \\
0
\end{array}\right] \tilde{d} \\
n & =\left[\begin{array}{ll}
1 & 0 \\
0 & 1
\end{array}\right] x_{d i}
\end{aligned}
$$

where $\sigma=-0.001$.

Let $x^{T}=\left[\begin{array}{llllllll}\ell \theta & \ell \psi & \ell \dot{\theta} & \ell \dot{\psi} & \phi_{\theta} & \phi_{\psi} & x_{d 1} & x_{d 2}\end{array}\right], w=\tilde{d}$ and $u^{T}=\left[\begin{array}{ll}e_{\theta} & e_{\psi}\end{array}\right]$. Combining rotor dynamics and the 
disturbance equation, the linearized equation can be written as

$$
\begin{aligned}
\dot{x} & =A(\rho) x+B_{1} w+B_{2} u \\
z & =C_{1} x+D_{11} w+D_{12} u \\
y & =C_{2} x+D_{21} w+D_{22} u
\end{aligned}
$$

with

$$
A=\left[\begin{array}{cccccccc}
0 & 0 & 1 & 0 & 0 & 0 & 0 & 0 \\
0 & 0 & 0 & 1 & 0 & 0 & 0 & 0 \\
-\frac{4 c_{2}}{m} & 0 & 0 & -\frac{\rho J_{a}}{J_{r}} & \frac{2 c_{1}}{m} & 0 & 0 & 0 \\
0 & -\frac{4 c_{2}}{m} & \frac{\rho J_{a}}{J_{r}} & 0 & 0 & \frac{2 c_{1}}{m} & 0 & 0 \\
\frac{2 d_{2}}{N} & 0 & 0 & 0 & -\frac{d_{1}}{N} & 0 & 0 & 0 \\
0 & \frac{2 d_{2}}{N} & 0 & 0 & 0 & -\frac{d_{1}}{N} & 0 & 0 \\
0 & 0 & 0 & 0 & 0 & 0 & \sigma & -\rho \\
0 & 0 & 0 & 0 & 0 & 0 & \rho & \sigma
\end{array}\right],
$$

$$
\begin{aligned}
& B_{1}=\left[\begin{array}{c}
0_{6 \times 1} \\
\mathbf{1} \\
0
\end{array}\right], \quad B_{2}=\frac{1}{N}\left[\begin{array}{c}
0_{4 \times 2} \\
I_{2} \\
0_{2 \times 2}
\end{array}\right] \\
& C_{1}=\left[\begin{array}{cc}
I_{2} & 0_{2 \times 6} \\
0_{2 \times 8}
\end{array}\right], \quad D_{11}=0_{4 \times 1}, \quad D_{12}=\left[\begin{array}{c}
0_{2 \times 2} \\
I_{2}
\end{array}\right],
\end{aligned}
$$

$C_{2}=\left[\begin{array}{lll}I_{2} & 0_{2 \times 4} & I_{2}\end{array}\right], \quad D_{21}=0_{2 \times 1}, \quad D_{22}=0_{2 \times 2}$

Note that the linearized rotor dynamic equation is in the form of a linear parameter-varying system with the rotor speed serving as the parameter. For gainscheduled control, the rotational speed is assumed to be available in real-time for controller use.

\section{Interpolating LPV Control Design}

The design objectives of the LPV controller are to asymptotically stabilize the system over the whole range of rotor speeds and to minimize an error signal representing a weighted sum of the forces at the bearings, the gap displacement at the bearing locations, and the control input used.

Although the scheduling variable is time-varying in the LPV dynamics, it is simply treated as a fixed parameter in the design stage. The design objective is quantified from a frozen parameter design viewpoint by weighting functions, and the weighted open-loop interconnection is given in Figure 1.

In Figure 1 the weighting functions are chosen as

$$
\begin{aligned}
W_{e}(s) & =\frac{200(s+100)}{s+0.01} I_{2}, \\
W_{u}(s) & =\frac{0.001 s}{0.05 s+1} I_{2}, \\
W_{n}(s) & =0.001
\end{aligned}
$$

The rotor speed is assumed to change freely between $315 \mathrm{rad} / \mathrm{s}$ to $1100 \mathrm{rad} / \mathrm{s}$. The rotor dynamics exhibit significant gyroscopic effects in this speed

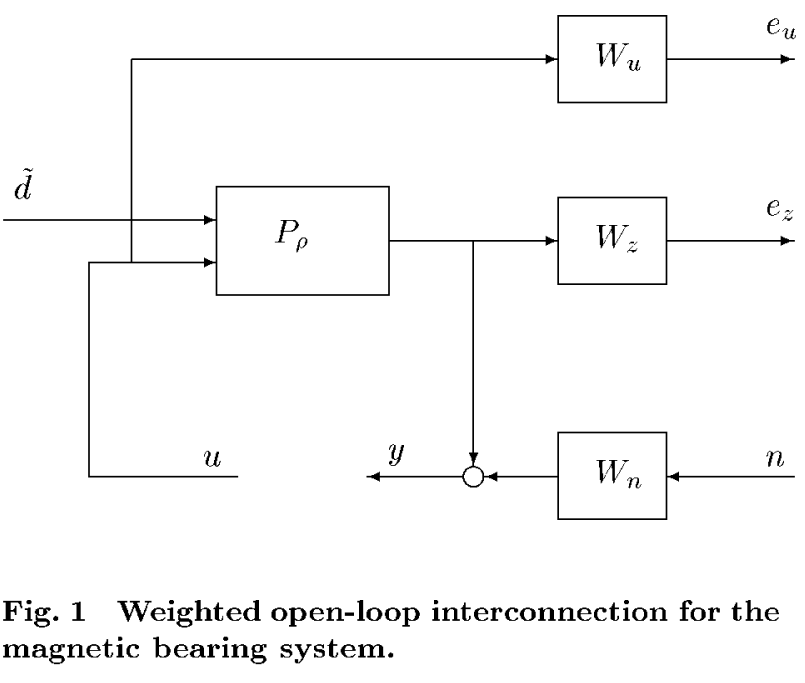

range. The LPV synthesis problem can be solved using either a single or parameter-dependent quadratic Lyapunov function over all gridding points in a onedimensional parameter space. ${ }^{5,8}$ The performance obtained through a single quadratic Lyapunov function (SQLF) approach is $\gamma=320.78$, which is quite conservative. Also, multiple LPV control syntheses are conducted with the whole parameter space divided into two subsets $([315,720] \cup[700,1100])$ and four subsets $([315,520] \cup[500,720] \cup[700,920] \cup[900,1100])$, respectively. Then five points are used to grid each parameter subspace uniformly. The free parameters $Q_{R}$ and $Q_{S}$ are chosen as $0.1 I_{12}$ for $\rho \in \mathcal{P}^{i} \cap \mathcal{P}^{j}$ or zero otherwise. In each partitioned parameter space case, two sets of identical basis functions are used to parameterize the functional space,

$$
\begin{aligned}
& f_{i}^{1}(\rho)=1, \\
& g_{i}^{1}(\rho)=1, \quad g_{i}^{2}(\rho)=\rho
\end{aligned}
$$

for each $i$. Note that the basis function for $R_{i}(\rho)$ is chosen as a constant over each parameter subset. This will only cause small performance degradation compared with more complicated basis function selections. Since both $R$ and $S$ become functions of scheduling parameters after interpolation, the interpolated LPV controller gain will depend on both the parameter and its derivative. The performance level of the interpolating LPV controller versus number of parameter subsets under different parameter variation rates is shown in Table 2. Note that the $\gamma$ value represents the "worst-case" interpolating LPV control performance over different parameter subsets. The actual performance over each parameter subset could be less than the "worst-case" performance.

For comparison, a single parameter-dependent Lyapunov function is also considered to demonstrate the sharpness of the newly proposed LPV synthesis condition. The calculated performance bound is about the same as the "worst-case" performance of interpolated LPV cases. 


\begin{tabular}{c|ccc}
\hline Variation rate & PDLF & \multicolumn{3}{|c}{ Interpolating LPV } \\
& & 2 subsets & 4 subsets \\
\hline 1 & 9.863 & 9.936 & 9.954 \\
100 & 10.064 & $\mathbf{1 0 . 1 0 7}$ & 10.149 \\
$\mathbf{1 0 0 0 0}$ & 141.24 & $\mathbf{1 4 4 . 4 3}$ & 143.50
\end{tabular}

Table 2 Achievable induced- $\mathcal{L}_{2}$ performance using single and multiple parameter-dependent Lyapunov functions (PDLFs).

\section{Simulation Results}

Next, the LPV controller synthesized using two subsets is used for comparison study and simulation work.

To compare the LPV controller with the optimal $\mathcal{H}_{\infty}$ controller, eight points are chosen from the parameter set $\mathcal{P}$ and $\mathcal{H}_{\infty}$ controller is synthesized for each operating condition. Then their $\mathcal{H}_{\infty}$ norm values are compared with the sub-optimal control performance that is achieved by LPV controllers evaluated at fixed parameter values. These are provided in the Table 3.

\begin{tabular}{c|cc}
\hline$\rho(\mathrm{rad} / \mathrm{s})$ & $\mathcal{H}_{\infty}$ & Interpolating LPV \\
\hline 315 & 9.778 & 9.833 \\
427.1 & 7.487 & 8.147 \\
539.3 & 6.149 & 6.719 \\
651.4 & 5.289 & 5.772 \\
763.6 & 4.696 & 4.988 \\
875.7 & 4.264 & 4.831 \\
987.9 & 3.936 & 4.600 \\
1100 & 3.678 & 4.383 \\
\hline
\end{tabular}

Table 3 Frozen optimal/LPV closed-loop $\mathcal{H}_{\infty}$ norm.

Since the LPV controller is designed for a range of parameters, it is not surprising that they are only suboptimal for each fixed parameter value. However, it is observed that the optimal performance level using $\mathcal{H}_{\infty}$ is very close to the achievable performance derived from switching the LPV controller at each frozen parameter. However, the optimal controller is highly tuned to its designated rotor speed. When a magnetic bearing operates at a different rotational speed, significant performance degradation or even loss of stability is expected. For example, the optimal $\mathcal{H}_{\infty}$ controller designed for $\rho=700 \mathrm{rad} / \mathrm{s}$ results in a performance level $\gamma=1.16 \times 10^{6}$ when the rotor is actually rotating with the speed of $1100 \mathrm{rad} / \mathrm{s}$.

Finally, we compare the closed-loop step response for both optimal and LPV controllers. The time responses for the optimal controller and the LPV controller are shown in Figures 2 and Figure 3, respectively. It can be seen that the step responses of rotor displacement from both controllers are quite similar, whereas the control actions are slightly different due to sub-optimality of LPV controllers.

After analyzing the frozen point LPV controller property, we are ready to do some nonlinear simula-
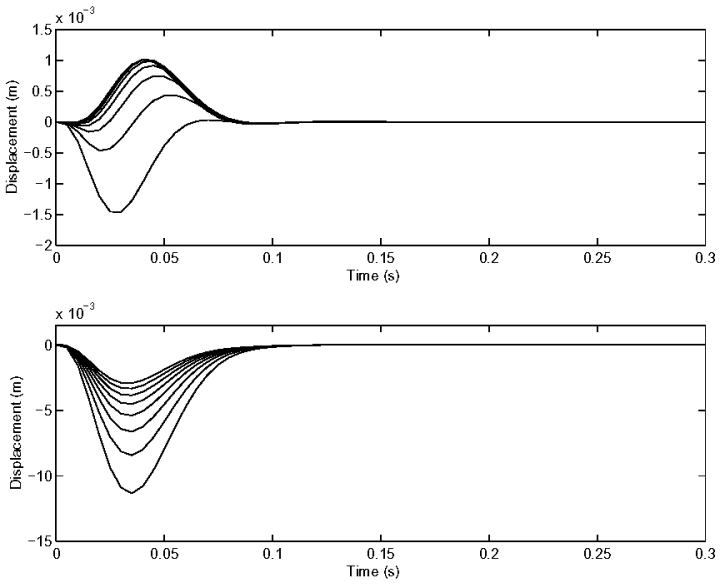

(a) rotor displacement $x_{1}, x_{2}$
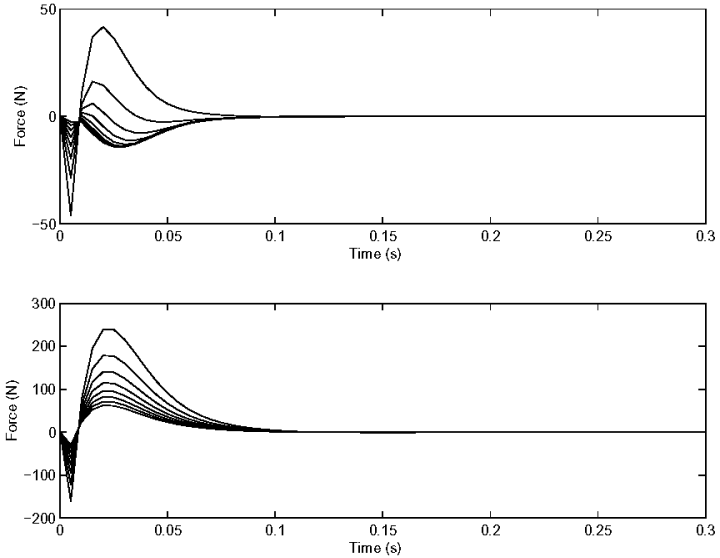

(b) control force $u_{1}, u_{2}$

Fig. 2 Fixed parameter unit-step response with $\mathcal{H}_{\infty}$ optimal controller.

tions for $\mathrm{AMB}$ with time-varying rotational speed. A time-varying rotor speed profile is chosen as

$$
d(t)= \begin{cases}\sin (680 t) & 0 \leq t<0.5 s \\ \sin (\omega t), \omega=655+50 t & 0.5 s \leq t<1.5 s \\ \sin (730 t) & 1.5 s \leq t<2 s\end{cases}
$$

Note that the rotor speed trajectory is deliberately chosen to cross the intersection of two subsets $[315,720]$ and $[700,1100]$ of the scheduling parameter to illustrate LPV controller interpolating effect. Disturbance $\tilde{d}=1.3 \times 10^{-5}$ is used for the simulation purpose. Except for small glitches during the controller interpolation period, the simulation results demonstrate good performance of interpolating LPV control as shown in Figure 4.

\section{Concluding Remarks}

In this paper, an LPV control interpolation algorithm was proposed to achieve high-performance of gain-scheduled control. The proposed LPV control approach unified the systematic LPV control theory with interpolation technique. The stability of the 

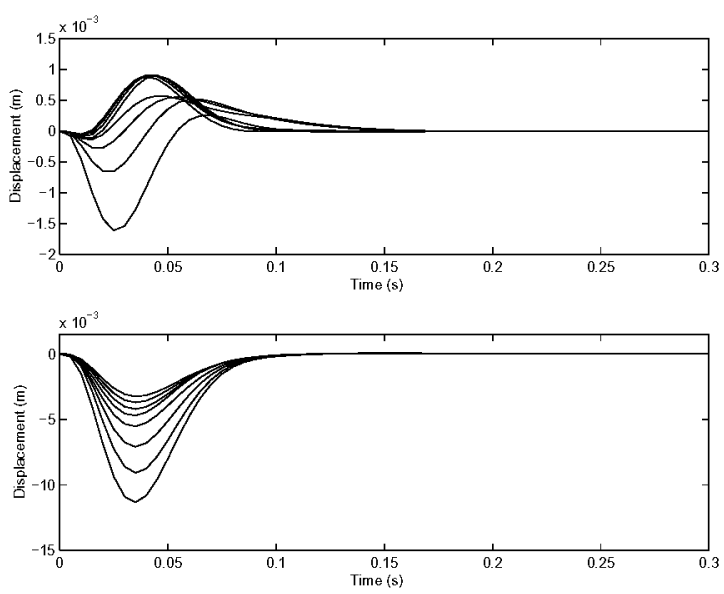

(a) rotor displacement $x_{1}, x_{2}$
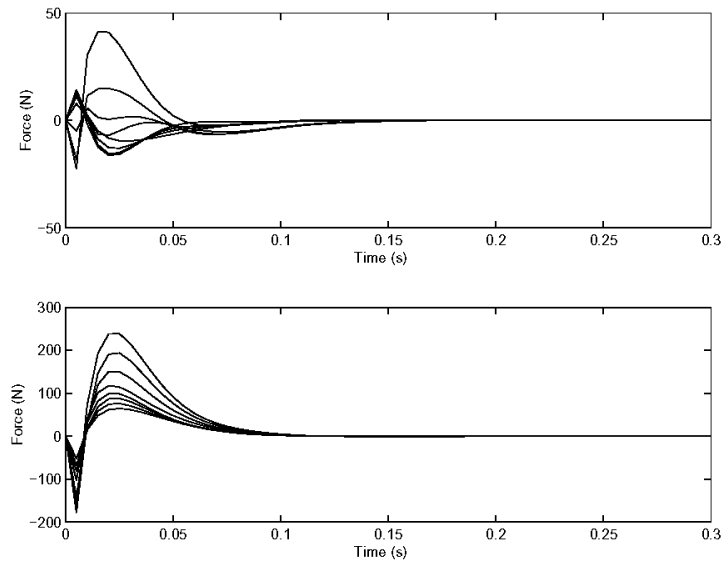

(b) control force $u_{1}, u_{2}$

Fig. 3 Fixed parameter unit-step response with LPV controller.

interpolated LPV control was achieved by enforcing continuity of Lyapunov function over entire parameter space. With modest increase of computational effort, the proposed LPV interpolation scheme improved the LPV control performance considerably. The newly developed LPV control synthesis method was applied to the magnetic bearing control problem to reject unbalancing sinusoidal disturbances and accommodate changing rotor speed. Promising simulation results were obtained.

For future research, an F-16 nonlinear model has been acquired from the NASA Langley Research Center and the proposed LPV control technique is currently being applied to a highly maneuverable aircraft to improve its performance over the expanded flight envelope into nonlinear unsteady flight regimes. Because aerodynamic controls may be insufficient for control at post-stall conditions, we would like to augment the conventional aerodynamic surface controls using thrust vectoring (TV) control effectors. The LPV interpolated control design approach is capable of unifying the aerodynamic force and thrust force control law development in a unified framework, and

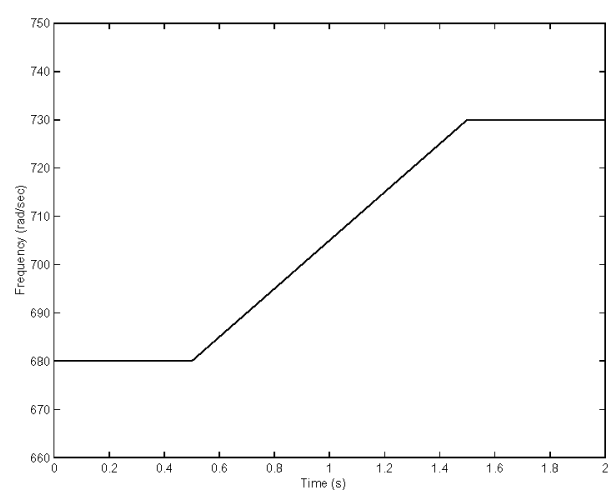

(a) Rotor speed profile

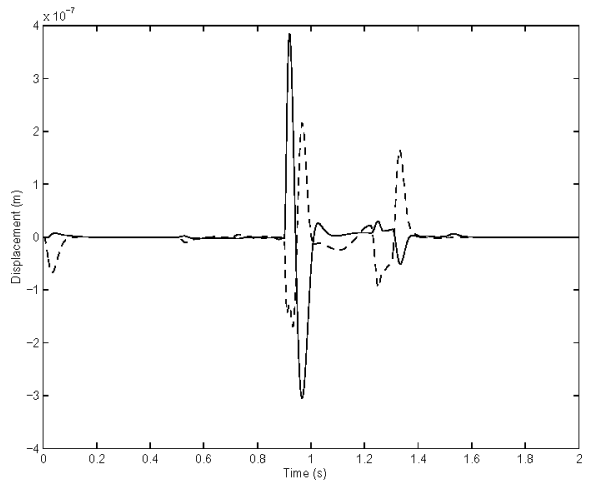

(b) Displacement: $x_{1}$-solid, $x_{2}$-dash

Fig. 4 Performance of interpolating LPV control for time-varying rotor speed.

of providing guaranteed stability and performance for large flight envelope.

\section{References}

${ }^{1}$ W.J. Rugh, "Analytical framework for gain scheduling," IEEE Contr. Sys. Mag., 11(1):74-84, 1991.

${ }^{2}$ J.S. Shamma and M. Athans, "Analysis of Gain Scheduled Control for Nonlinear Plants," IEEE Trans. Automat. Contr., AC-35:898-907, 1990.

${ }^{3}$ P. Apkarian and R.J. Adams, "Advanced Gain-scheduling Techniques for Uncertain Systems," IEEE Trans. Contr. Syst. Tech., 6(1):21-32, 1997.

${ }^{4} \mathrm{P}$. Apkarian and P. Gahinet, "A Convex Characterization of Gain-scheduled $\mathcal{H}_{\infty}$ Controllers," IEEE Trans. Automat. Contr., AC-40(9):853-864, 1995 .

${ }^{5}$ G. Becker and A.K. Packard, "Robust Performance of Linear Parametrically Varying Systems using Parametrically Dependent Linear Dynamic Feedback," Syst. Contr. Letts., $\mathbf{2 3}(3): 205-215,1994$.

${ }^{6}$ A.K. Packard, "Gain Scheduling via Linear Fractional Transformations," Syst. Contr. Letts., 22(2):79-92, 1994.

${ }^{7}$ C.W. Scherer, "Robust Mixed Control and LPV Control with Full Block Scalings," in Recent Advances of LMI Methods in Control (L. El Ghaoui, S. Niculescu ed.), SIAM, 1999.

${ }^{8}$ F. Wu, X.H. Yang, A.K. Packard, and G. Becker. "Induced $\mathcal{L}_{2}$ Norm Control for LPV Systems with Bounded Parameter Variation Rates," Int. J. Robust Non. Contr., 6(9/10):983-998, 1996.

${ }^{9}$ D.J. Stilwell and W.J. Rugh, "Stability Preserving Interpolation Methods for the Synthesis of Gain Scheduled Controllers," Automatica, 36:665-671, 2000.

${ }^{10} \mathrm{~S}$. Lim and J.P. How, "Control of LPV systems using a 
Quasi-Piecewise Affine Parameter-Dependent Lyapunov Function," in Proc. Amer. Contr. Conf., 1998, pp. 1200-1204.

${ }^{11}$ J.B. Rose, "An Electro-Mechanical Energy Storage System for Space Application," in Progress in Astronautics and Rockery, 3:613-622, 1961.

${ }^{12}$ C.D. Hall, "High Speed Flywheels for Integrated Energy Storage and Attitude Control," in Proc. Amer. Contr. Conf., 1997, pp. 1894-1898.

${ }^{13}$ M.-R. Nam, T. Hashimoto, and K. Ninomiya, "Design of $\mathcal{H}_{\infty}$ Attitude Controllers for Spacecraft using A Magnetically Suspended Momentum Wheel," Euro. J. Contr., 3:114-124, 1997.

${ }^{14} \mathrm{P}$. Tsiotras, H. Shen and C. Hall, "Satellite Attitude Control and Power Tracking with Momentum Wheels," in Proc. AAS/AIAA Astrodynamics Specialist Conf., AAS paper No. 99$317,1999$.

${ }^{15}$ A.M. Mohamed and I. Busch-Vishmiac, "Imbalance Compensation and Automation Balancing in Magnetic Bearing Systems using the $Q$-parameterization Theory," IEEE Trans. Contr. Sys. Tech., 3(2):202-211, 1995.

${ }^{16} \mathrm{~F}$. Matsumura, T. Namerikawa, K. Hagiwara, and M. Fujita, "Application of Gain Scheduled $\mathcal{H}_{\infty}$ Robust Controllers to A Magnetic Bearing," IEEE Trans. Contr. Sys. Tech., 4(5):484$493,1996$.

${ }^{17} \mathrm{~K}$. Nonami and T. Ito, " $\mu$ Synthesis of Flexible Rotormagnetic Bearing System," IEEE Trans. Contr. Sys. Tech., 4(5):503-512, 1996.

${ }^{18} \mathrm{P}$. Tsiotras and S. Mason. "Self-scheduled $\mathcal{H}_{\infty}$ Controllers for Magnetic Bearings," in Proc. ASME Int. Mech. Eng. Congress Expo., 1996, pp. 151-158.

${ }^{19} \mathrm{P}$. Tsiotras and C. Knospe, "Reducing Conservatism for Gain-scheduled $\mathcal{H}_{\infty}$ Controllers for AMB's," in Proc. MAG' 97 Magnetic Bearings Ind. Conf. Exhib., 1997.

${ }^{20} \mathrm{~S}$. Sivrioglu and K. Nonami, "LMI Approach to Gain Scheduled $\mathcal{H}_{\infty}$ Control Beyond PID Control for Gyroscopic Rotor-Magnetic Bearing Systems," in Proc. 35th IEEE Conf. Dec. Contr., 1996, pp. 3694-3699. 